\title{
Ten genes and two topologies: an exploration of higher relationships in skipper butterflies (Hesperiidae)
}

\author{
Ranjit Kumar Sahoo ${ }^{\text {Corresp.. }}{ }^{1}$, Andrew D. Warren ${ }^{2}$, Niklas Wahlberg ${ }^{3,4}$, Andrew V. Z. Brower ${ }^{5}$, Vladimir A \\ Lukhtanov $^{6}$, Ullasa Kodandaramaiah ${ }^{1}$ \\ ${ }^{1}$ School of Biology, Indian Institute of Science Education and Research Thiruvananthapuram, Thiruvananthapuram, Kerala, India \\ 2 McGuire Center for Lepidoptera and Biodiversity, Florida Museum of Natural History, University of Florida, PO Box 112710, 3215 Hull Rd., UF Cultural \\ Plaza, Gainesville, Florida 32611-2710, United States \\ 3 Department of Biology, Lund University, Lund, Sweden \\ 4 Department of Biology, University of Turku, Turku, Finland \\ 5 Evolution and Ecology Group, Department of Biology, Middle Tennessee State University, Murfreesboro, Tennessee, United States \\ 6 Department of Insect Systematics, Zoological Institute of Russian Academy of Sciences, Universitetskaya nab, St. Petersburg, Russia \\ Corresponding Author: Ranjit Kumar Sahoo \\ Email address: sahoork@iisertvm.ac.in
}

Despite multiple attempts to infer the higher level phylogenetic relationships of skipper butterflies (Family Hesperiidae), uncertainties in the deep clade relationships persist. The most recent phylogenetic analysis included fewer than 30\% of known genera and data from three gene markers. We here reconstruct the higher-level relationships with a rich sampling of ten nuclear and mitochondrial markers ( 7726 base pairs) from 270 genera and find two distinct but equally plausible topologies among subfamilies at the base of the tree. In one set of analyses, the nuclear markers suggest two contrasting topologies, one of which is supported by the mitochondrial dataset. However, another set of analyses suggests mito-nuclear conflict as the reason for topological incongruence. Neither topology is strongly supported, and we conclude that there is insufficient phylogenetic evidence in the molecular dataset to resolve these relationships. Nevertheless, taking morphological characters into consideration, we suggest that one of the topologies is more likely. 
1 Title: Ten genes and two topologies: an exploration of higher relationships in 2 skipper butterflies (Hesperiidae).

5 Authors: Ranjit Kumar Sahoo ${ }^{1}$, Andrew D. Warren², Niklas Wahlberg ${ }^{3,4}$, Andrew V. Z.

6 Brower $^{5}$, Vladimir A. Lukhtanov ${ }^{6}$, Ullasa Kodandaramaiah ${ }^{1}$

$9{ }^{1}$ School of Biology, Indian Institute of Science Education and Research Thiruvananthapuram, 10 Kerala 695016, India.

$12{ }^{2} \mathrm{McGuire}$ Center for Lepidoptera and Biodiversity, Florida Museum of Natural History, 13 University of Florida, PO Box 112710, 3215 Hull Rd., UF Cultural Plaza, Gainesville, FL $14 \quad 32611-2710$ USA

${ }^{3}$ Department of Biology, University of Turku, 20014 Turku, Finland

${ }^{4}$ Department of Biology, Sölvegatan 37, Lund University, 22362 Lund, Sweden

19 Evolution and Ecology Group, Department of Biology, Middle Tennessee State University, 20 Murfreesboro, TN 37037 USA

${ }^{6}$ Department of Insect Systematics, Zoological Institute of Russian Academy of Sciences,

23 Universitetskaya nab. 1, 199034 St. Petersburg, Russia

25 Corresponding Author: 
26

27 School of Biology, Indian Institute of Science Education and Research Thiruvananthapuram,

28 Kerala 695016, India.

29

$\underline{\text { sahoork@iisertvm.ac.in }}$

Ranjit Kumar Sahoo ${ }^{1}$
30

31

32

33

34

35

36

37

38

39

40

41

42

43

44

45

46

47

51

52

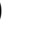

\section{Abstract:}

Despite multiple attempts to infer the higher level phylogenetic relationships of skipper butterflies (Family Hesperiidae), uncertainties in the deep clade relationships persist. The most recent phylogenetic analysis included fewer than $30 \%$ of known genera and data from three gene markers. We here reconstruct the higher-level relationships with a rich sampling of ten nuclear and mitochondrial markers (7726 base pairs) from 270 genera and find two distinct but equally plausible topologies among subfamilies at the base of the tree. In one set of analyses, the nuclear markers suggest two contrasting topologies, one of which is supported by the mitochondrial dataset. However, another set of analyses suggests mito-nuclear conflict as the reason for topological incongruence. Neither topology is strongly supported, and we conclude that there is insufficient phylogenetic evidence in the molecular dataset to resolve these relationships. Nevertheless, taking morphological characters into consideration, we suggest that one of the topologies is more likely. 
56

57

58

59

60

61

62

63

64

65

66

67

68

69

70

71

72

73

74

75

76

77

78

PeerJ reviewing PDF | (2016:05:10878:2:0:NEW 30 Sep 2016) 
Introduction:

A robust phylogeny is the key to understanding historical macroevolutionary processes that have shaped extant diversity. For instance, a phylogenetic hypothesis is needed to address questions regarding patterns of morphological evolution, coevolution, and historical biogeography, as well as for a higher-level classification system. Among invertebrates, butterflies have been the most popular study systems in evolutionary biology (Boggs et al. 2003). Relationships among and within butterfly families have been largely studied by phylogenetic analyses of DNA sequence data (Campbell, Brower \& Pierce, 2000; Caterino et al., 2001; Braby, Vila \& Pierce, 2006; Nazari, Zakharov \& Sperling, 2007; Wahlberg et al., 2009; Simonsen et al., 2011; Heikkila et al., 2012; Wahlberg et al., 2014; Espeland et al., 2015). Yet, the higher level relationships among skipper butterflies, with more than 4000 species in about 567 genera (Warren, Ogawa \& Brower, 2008) and representing a fifth of the world's butterfly fauna (Hernández-Roldán, Bofill \& Dapporto, 2014), are still unsatisfactorily resolved.

102

Until recently, the higher-level classification of the family that has been generally followed was that proposed by Evans (1949) based on morphological characters. However, a major problem with skipper systematics is the remarkable uniformity of morphological structure among skipper taxa, which makes phenotype-based grouping extremely challenging (Voss, 1952; Warren,

107 Ogawa \& Brower, 2008). Following multiple attempts over the last several decades (Voss, 1952;

108 Ackery, 1984; Scott, 1985; Scott and Wright, 1990; Chou, 1994, 1999; Mielke, 2005), a recent 109 study employing molecular data suggested a classification that included five subfamilies (Warren, 110 Ogawa \& Brower, 2008). This classification relied on analyses of one mitochondrial and two 
111 nuclear markers, a dataset of 2085 bp (base pairs). A subsequent analysis that added

112 morphological data (49 characters) to the same molecular data led to a revised classification that

113 included seven subfamilies (Warren, Ogawa \& Brower, 2009).

114

115 Warren and colleagues (Warren, Ogawa \& Brower, 2008; Warren, Ogawa \& Brower, 2009) used

116 Maximum Parsimony analyses with nodal support estimated through Bremer Support values

117 (Bremer, 1994). Despite some strongly supported monophyletic taxa being recovered, many

118 putative higher clades were unresolved. Specifically, uncertainty remained about relationships

119 among the major clades within the subfamily Pyrginae. Furthermore, support for relationships

120 among the monophyletic subfamilies Heteropterinae, Trapezitinae and Hesperiinae was weak to

121 moderate. The status of Euschemoninae as sister to rest of the family, except Coeliadinae,

122 received very low nodal support (Bremer support $=1$ ), although this placement is corroborated

123 by the early developmental characters of Euschemoninae, which are similar to those of

124 Coeliadinae and Eudaminae (Warren, Ogawa \& Brower, 2009).

125

126 Yuan et al. (2015) investigated relationships among a small subset of hesperiid taxa - 23 genera

127 from China - using 1458 bp of mitochondrial sequence data. Their Maximum Likelihood tree

128 also indicated uncertainty in the position of Eudaminae and Pyrginae. Another study based on

129 complete mitochondrial genomes of six skipper butterflies representing five subfamilies (sensu

130 Warren, Ogawa \& Brower, 2009) failed to support the monophyly of Pyrginae (Kim et al., 2014).

132 In summary, existing studies on the higher-level relationships within this speciose butterfly 133 family have indicated significant conflicts (Warren, Ogawa \& Brower, 2008; Warren, Ogawa \& 134 Brower, 2009; Kim et al., 2014; Yuan et al., 2015), and we currently lack a robust higher level 135 phylogenetic hypothesis for evolutionary studies or a sub-family level classification. The reasons 136 for conflicting topologies across studies and poor nodal support could be a) incongruence among 137 gene trees due to incomplete lineage sorting (Pollard et al., 2006; Whitfield \& Lockhart, 2007), b) 138 ancestral introgression (Eckert \& Carstens, 2008), c) differences in characteristics of the datasets 139 used (Nabholz et al., 2011), d) poor taxon sampling, e) insufficient data to resolve deeper nodes 140 (Wolf et al., 2002; Rokas et al., 2003) or, f) a near-hard polytomy due to a rapid-radiation 141 (Kodandaramaiah et al., 2010).

143 In order to bring further understanding to the higher-level phylogeny of skipper butterflies, we 144 assembled sequences of ten gene regions from 270 genera and analyzed a 7726 bp dataset using 145 both parsimony and model-based tree reconstruction methods. We also compiled the complete 
146 mitochondrial genome of 15 skipper species across five subfamilies from GenBank to compare

147 the tree from the mitochondrial genome with that of single mitochondrial and combined-nuclear 148 genes. Consistent with the existing conflict across studies (Warren, Ogawa \& Brower, 2008; 149 Warren, Ogawa \& Brower, 2009; Kim et al., 2014; Yuan et al., 2015), our analyses showed 150 conflicting topologies at the deeper nodes of the phylogeny. To understand the reasons for the 151 uncertainty in the phylogenetic estimation, we followed an integrative approach with systematic 152 data encoding and tree comparison.

Methods:

\section{Taxon and gene sampling:}

157 Our analyses were based on 311 ingroup specimens representing 270 hesperiid genera and 12 158 outgroup taxa (5 Papilionidae, 2 Hedylidae and 5 Pieridae). This dataset builds on the previous 159 study by Warren, Ogawa \& Brower (2009) that included sequences of three protein-coding genes 160 (mtDNA COI, EF1a and wingless). We sequenced an additional part of COI and seven more 161 genes (ArgKin, CAD, GAPDH, IDH, MDH, RpS2 and RpS5) using protocols and primers from 162 Wahlberg \& Wheat (2008). A new primer-pair was designed (Table S1) to amplify the gene IDH 163 for certain taxa. We have also included 96 additional specimens representing 71 genera to the 164 present analyses. Our taxon sampling accounts for $60-70 \%$ of the genera of Coeliadinae (6 165 genera), Eudaminae (38 genera), Heteropterinae (7 genera), and Trapezitinae (11 genera); 40-50 $166 \%$ of Pyrginae (73 genera) and Hesperiinae (134 genera), and $100 \%$ of Euschemoninae (1 167 genus). The sequences for outgroups were acquired from GenBank. We included the 168 morphological, behavioral and ecological data matrix used in Warren, Ogawa \& Brower (2009) 169 in certain analyses. The sequences generated during this study were deposited in GenBank. The 170 molecular data matrix in our study comprised 7726 characters, more than three times that of the 171 previous dataset (Warren, Ogawa \& Brower, 2008; Warren, Ogawa \& Brower, 2009).

\section{Dataset encoding:}

174 Along with the analysis of the concatenated dataset (nt_123), we generated context specific 175 datasets from the concatenated gene matrix for various analyses designed to identify potential 176 sources of conflict and/or poor nodal support. In one analysis, accounting for the impact of 177 compositional heterogeneity, we assigned ambiguity to all the sites that potentially experience 178 synonymous change (degen_1) (Regier et al., 2010; Zwick et al., 2012). We also checked the 179 extent of substitution saturation in each gene matrix using DAMBE v6.4.20 (Xia, 2013), which 180 showed saturation in $3^{\text {rd }}$ codon positions (Figure S1). To account for substitution saturation and 
181 degeneracy, we removed the $3^{\text {rd }}$ codon positions and the $1^{\text {st }}$ codon positions coding for Arginine

182 or Lysine (noLRall1+nt2) (Regier et al., 2008). In further analyses, we removed the $3^{\text {rd }}$ coding

183 positions from the concatenated dataset (nt_12) or only from the mitochondrial genes

184 (nuclear_123+CO_12).

185

186 We also analyzed all the nuclear genes together (nuclear_123) and reconstructed multi-gene and single gene trees for comparison. In subsequent analyses, we also combined the morphological, ecological and behavioral characters from Warren, Ogawa \& Brower (2009) with certain molecular datasets - nt_123 and nuclear_123. In addition, we analysed an assembly of all protein coding sequences (13 genes) from the mitochondrial genomes of 15 skipper butterflies acquired from GenBank.

Phylogenetic analyses:

We performed Maximum Parsimony analyses in TNT v.1.1 (Goloboff, Farris \& Nixon, 2008) using 'New Technology' searches (Goloboff, 1999; Nixon, 1999) (consisting of tree fusion, sectorial search, ratchet and tree drift) with 1000 random addition replicates; nodal supports were derived from 1000 bootstrap replicates (Felsenstein, 1985). For Maximum Likelihood (ML) and Bayesian Inference (BI) analyses, we used RAxML v8 (Stamatakis, 2014) and MrBayes v3.2 (Ronquist et al., 2012) respectively, on the XSEDE web server through the CIPRES Science gateway (Miller, Pfeiffer \& Schwartz, 2010). For ML analyses, we used the GTR model of substitution with gamma model of rate heterogeneity $(\mathrm{GTR}+\mathrm{G})$ and different partition schemes,; either gene-based or based on rates of evolution calculated by the program TIGER (Cummins \& McInerney, 2011). In gene-based partitions each gene was considered as a separate partition, while in TIGER-partitions, the characters were binned together based on their rate of evolution regardless of gene origin (used as a partitioning strategy in Rota \& Wahlberg, 2012). TIGERpartitions for the dataset were derived from the program TIGER (Tree Independent Generation of Evolutionary Rates) that calculates relative rates of evolution of each site in an alignment (Cummins \& McInerney, 2011). The data were then divided into seven partitions based on the relative rates using an algorithm developed by Tobias Malm (J. Rota, T. Malm \& N Wahlberg, in prep.) such that the first partition consisted of the invariant and very slowly evolving sites and the last partition consisted of sites evolving very quickly. To check whether model selection had

212 any impact on the tree reconstruction, we also performed the above ML analyses using the best

213 fitted model from PartitionFinder v1.1.1 (Lanfear et al., 2012) (detail in Figure S2). For each ML

214 analyses, the node supports were computed from 1000 bootstrap replicates. Single gene matrices

215 were analyzed with $\mathrm{GTR}+\mathrm{G}$ model and the node supports were derived from 500 bootstrap

216 replicates. For single gene analyses, we dropped the taxa for which the corresponding gene

217 sequence was not available. For the mitogenome analysis, we performed ML tree searches with 
218 codon-based partitions and estimated the nodal support from 100 bootstraps.

220

221

222

223

224

225

226

227

228

229

230

231

232

233

234

235

236

237

238

239

240

241

242

243

244

245

246

247

248

249

250

251

The BI analysis of the concatenated dataset with TIGER-partitions was performed with a mixed model of substitution which samples all possible models in the GTR family in proportion to their posterior distributions (Huelsenbeck et al., 2004) as implemented in MrBayes 3.2 (Ronquist et al., 2012). We assigned the gamma model of rate heterogeneity to all the partitions; the first partition was additionally assigned a proportion of invariable sites. The program MrBayes was set to estimate the base frequencies and shape parameters from the data. Two independent runs with two chains per run were performed for $\sim 30$ million generations, sampling trees every 10000 generations. The convergence of independent runs was analyzed from the values of potential scale reduction factors (PSRF) (PSRF close to 1 determines convergence) (Gelman \& Rubin, 1992); we also checked the plots of log-likelihoods and other parameters on Tracer v1.6 (Rambaut et al., 2014).

\section{Tree comparison:}

To investigate the differences among the trees from multiple analyses, we compared the trees (except single gene trees) for their topological incongruence with and without the likelihood scores. While the non-likelihood incongruence test reflects differences in branching patterns among the topologies, the likelihood-based comparison calculates the difference between competing hypotheses with distinct topologies for a given dataset (Planet, 2006). For the nonlikelihood based tree comparison at the deep level divergences, we used a Lento plot to depict the conflict among different trees in a two-dimensional graph (Lento et al., 1995). We employed the approximate unbiased (AU) test (Shimodaira, 2002) for likelihood-based tree comparisons. To check for incongruence among gene trees, we used partitioned bremer support (PBS) analysis that examines the contribution of each gene partition to the topological support of the consensus tree (Baker \& DeSalle, 1997).

\section{Results:}

\section{Multilocus tree estimation:}

Trees from the concatenated dataset, irrespective of parsimony or ML analysis or the partition scheme used, showed identical relationships among the early branches representing the major clades (sensu Warren, Ogawa \& Brower, 2009) (Figure 1A) - (i) Coeliadinae was sister to the rest of the family, (ii) Pyrginae was paraphyletic, (iii) Euschemoninae was sister to Eudaminae, 
252 and (iv) Heteropterinae, Trapezitinae and Hesperiinae were all monophyletic. The topology

253 remained unchanged even after the addition of morphological characters to the concatenated

254 dataset.

255

256

257

258

259

260

261

262

263

264

265

266

267

268

269

270

271

272

273

274

275

276

277

278

279

280

281

282

283

284

However, the ML trees from the combined-nuclear dataset (nuclear_123) showed a contrasting topology (Figure 1B) to that of the concatenated dataset, irrespective of the partitioning scheme: Pyrginae was monophyletic and Euschemoninae was sister to rest of Hesperiidae except Coeliadinae. The topology remained unchanged with the addition of morphological characters to the dataset (nuclear_123+morph) as well as analyses of the subsets of the combined-nuclear matrix (7gene_123, 8gene_123).

These dataset specific variation in tree topologies were consistent across different evolutionary model and were also found in partitioning scheme from PartitionFinder (detail in Figure S2).

ML analyses of non-degenerated datasets (nt_12, degen_1, noLRall1+nt2) resulted in unresolved tree topologies indicating insufficient phylogenetic signal in the $1^{\text {st }}$ and $2^{\text {nd }}$ codon positions of the dataset. (Figure 1C).

$\mathrm{BI}$ analysis of the concatenated dataset showed a topology similar to that of Figure 1B with a few changes (detail in Figure S3). Randomly sampled 100 trees from the MCMC generations, after discarding burnin, showed the presence of only one topology (as in Figure 1B); however, a very low proportion (6\%) of an alternate topology (as in Figure 1A) was found in one of the runs.

\section{Tree Comparison:}

To test whether multiple tree topologies across ML analyses are equally likely given the datasets, we performed an approximate unbiased (AU) test (Shimodaira, 2002) for both the concatenated and combined-nuclear datasets independently. The AU test, without any partitioning scheme, rejected $(p<0.0001)$ the tree topologies from the non-degenerate datasets (nt_12, degen, noLRall1+nt_2). Hence, we dropped the trees from non-degenerate data sets from further analyses of tree topological similarity at higher taxonomic levels. However, two distinct topologies (Figure 1A and 1B) were accepted as significant trees $(\mathrm{P}>0.05)$ for the combinednuclear dataset, whereas only one topology (Figure 1A) was significant $(\mathrm{P}>0.05)$ for the 
285

286

287

288

289

290

291

292

293

294

295

296

297

298

299

300

301

302

303

304

305

306

307

308

309

310

311

312

314

314

315

316

317

concatenated dataset.

A visual comparison of the two distinct tree topologies (Figure 1A and 1B) showed that all the subfamily clades (sensu Warren, Ogawa \& Brower, 2009) except Pyrginae were monophyletic (BS $>98)$. Pyrginae was recovered either as monophyletic $(\mathrm{BS}=7-60)$ or paraphyletic. Similarly, Euschemoninae and Eudaminae were either sisters $(\mathrm{BS}=58-76)$ or non-sisters. However, due to the presence of low BS values $(<76)$ in 6 out of 13 deep nodes across the analyses, we were uncertain whether there existed significant conflict among different tree topologies.

\section{Mito-genomic analysis:}

The ML tree from all 13 protein coding genes from complete mitochondrial genomes of 15 skipper butterflies had a tree-wide average BS of 80 (see Figure S4). Along with many wellsupported nodes (BS>97), low support values (BS $30-60)$ were also obtained for 5 out of 14 internal nodes. The lowest BS (=32) was obtained for the node containing Eudaminae, the lineages of Pyrginae and the common ancestor of Heteropterinae and Hesperiinae. The node that placed Eudaminae as sister to one of the Pyrginae clades had BS=42.

We found that the tree topology from the mitogenomic analysis corroborated the deep splits found in the COI gene tree; Eudaminae nested within Pyrginae rendering the latter paraphyletic, which was also the case for the concatenated dataset.

\section{Multiple plausible tree topologies:}

The presence of multiple tree topologies was not limited to the dataset-specific analyses. Regardless of the partitioning scheme or the dataset (concatenated and combined-nuclear dataset) used, almost equal numbers of contrasting tree topologies were present among the ML-bootstrap trees across multiple analyses (see Figure S5); hence, it is likely that the topology of the best ML tree is one among two equally likely topologies.

To investigate this further, we extracted 105 model-optimized trees from each of the ML analyses of the concatenated (nt_123) and combined-nuclear (nuclear_123) data, separately from datasets with both partitioning schemes (gene-partitions and TIGER-partitions). The clades in the resulting trees were collapsed to the subfamily level except Pyrginae, which was collapsed to the level of tribes (sensu Warren, Ogawa \& Brower, 2009), and were plotted on a Lento plot (Figure 
318 2) to check for support and conflict for each split. We observed conflicting splits among the trees 319 from the combined-nuclear dataset with TIGER-partitions, which indicated the presence of

320

321

322

323

324

325

326

327

328

329

330

331

332

333

334

335

336

337

338

339

340

341

342

343

344

345

346

347

348

349

350

351

352

353

354 multiple topologies; however, trees from the concatenated dataset had no conflicting splits, indicating a single topology. But the case was different when gene-partitions were used multiple topologies resulted from concatenated dataset and only one of these topologies was recovered from the combined-nuclear dataset. The AU test showed equal tree likelihoods ( $\mathrm{p}>$ 0.05) for multiple topologies for both concatenated and combined-nuclear datasets given the respective partition schemes as explained above.

Thus, we found two contrasting tree topologies, respectively supporting (i) monophyly of Pyrginae and non-sister status of Eudaminae and Euschemoninae, and (ii) paraphyly of Pyrginae and sister status of Eudaminae and Euschemoninae. In addition, we noticed that the position of Eudaminae with respect to Pyrginae varied across these contrasting topologies. While Eudaminae was sister to all Hesperiidae except Coeliadinae and Euschemoninae in the former, the latter topology indicated that Eudaminae was sister to the clade containing Heteropterinae, Trapezitinae and Hesperiinae.

We observed that the contrasting topologies had short branch lengths at the fluctuating clades: the interrelationship among Eudaminae, the major clades of Pyrginae and the common ancestor of Heteropterinae, Trapezitinae and Hesperiinae. The association of very low BS $(<60)$ with these short branches suggests possible topological conflict among gene trees. To investigate this hypothesis, we examined the relationships among the conflicting clades in the individual gene trees. Because the single gene trees had very low BS values for most of the clades, we clustered gene markers based on their topological congruency for the deeper clades and reanalyzed, expecting an improvement of nodal support and consistency in signal. For example, we combined all those gene markers from which Pyrginae was recovered as monophyletic and expected an improved BS for the Pyrginae clade in the gene-cluster analysis. As expected, this gene-cluster recovered Pyrginae as monophyletic with higher node support $(B S=80)$. However, none of the gene-clusters recovered the non-sister status of Eudaminae and Euschemoninae even though their sister status was poorly supported in all the gene-cluster analyses (Figure S6). This pattern of incongruence is not an artifact of missing data in our dataset, because sequential removal of taxa with $80-40 \%$ of missing data from the analyses changed neither the topology nor the support values at deeper nodes (Table S2); however, such sequential removal gradually reduced the proportion of alternate tree topologies in the tree set from best ML tree search (Figure S7). Hence, we could not accept the hypothesis that the two contrasting tree topologies are due to incongruence in gene histories; this is also evident from PBS analysis where no pattern of incongruence among gene trees were observed (Figure S8). 
Discussion:

357

358

359

360

361

362

363

364

365

366

367

368

369

370

371

372

373

374

375

376

377

378

379

380

381

382

383

384

385

386

387

388

389

With a dataset of $7726 \mathrm{bp}$ from 270 hesperiid genera, we present the most comprehensive phylogeny of this important group of butterflies. Our analyses suggested that there are two contrasting topologies for the higher-level skipper phylogeny. First, we reconstructed the phylogenetic trees using the concatenated and combined-nuclear datasets; the resulting trees were well-supported for higher-level relationships except at certain deep nodes. Tree comparisons revealed that there are multiple tree topologies for the relationships among major skipper lineages. We explicitly investigated gene-specific signals for the relationships among major clades, clustered them based on their topological congruence and reanalyzed to check for consistency.

\section{Conflicting topologies?}

Our analyses indicate the occurrence of two equally likely deep tree topologies (Figure 1A and 1B). Interestingly, the proximate reasons for the occurrence of these contrasting topologies appear to vary depending on the partitioning scheme used for the analysis. However, neither topology was strongly supported in any analysis and the results from our explorations of incongruence among gene histories were not conclusive. Gene-cluster analyses improved the nodal support for the monophyly of Pyrginae but were unable to recover the sister status of Eudaminae and Euschemoninae with good support. Similarly, from the PBS analysis, no pattern of incongruence in gene histories was observed. We conclude that there is insufficient information in the molecular dataset to resolve these relationships despite the extensive taxonomic sampling and large number of molecular characters.

The presence of conflicting topologies has also been reported from many other studies across plants (Soltis et al., 2002; Burleigh and Mathews 2004; Ruhfel et al., 2014) and animals (Rokas et al., 2003; Song et al., 2012). The possible reasons for topological incongruency are phylogenetic noise or conflict among gene trees (Smith et al., 2015). In case of the former, a concatenation approach is expected to give a better result (Rokas et al., 2003; Smith et al., 2015). In the latter case, where the conflict is presumed to be a result of gene flow across taxa or incomplete lineage sorting, coalescence based methods have been used for tree reconstruction (Jarvis et al., 2014; Xi et al., 2014; Smith et al., 2015). However, all there was very low node supports across gene trees, indicating that strong conflict across genes does not explain the patterns found here. We predict that a phylogenomic approach would provide a better outlook to 
390 this conflicting scenario or resolve the phylogeny, as such an approach has proved instrumental

391 in other studies (Dunn et al., 2008; Kocot et al., 2011; Smith et al., 2011; Johnson et al., 2013;

392 Jarvis et al., 2014; Richart et al., 2016).

393

394

395

396

397

398

399

400

401

402

403

404

405

406

407

408

409

410

412

413

414

415

416

417

418

419

420

421

422

423 We observed that the genus Cabirus, previously included within Eudaminae, grouped within 424 Achlyodini (subfamily Pyrginae). Further study of the morphology of Cabirus is needed to 425

\section{Systematic implications:}

Our study confirmed that all the subfamilies, possibly except Pyrginae, are monophyletic and received high BS support across multiple analyses. We are uncertain about the monophyly of Pyrginae, as our study reveals homoplastic character distributions that could potentially be explained by the occurrence of ancestral introgression among its early lineages. Hence, when a certain combination of genes was used for phylogenetic construction, Pyrginae was recovered monophyletic (e.g., Figure 1B), as in the previous study (Warren, Ogawa \& Brower, 2009). This result appears to be supported by morphology. Similarly, we remain uncertain about the true relationships among Eudaminae, Pyrginae and the clade containing Heteropterinae, Trapezitinae and Hesperiinae, due to short branches that may be explained by their rapid divergence from each other and possibly an introgression between Eudaminae and Euschemoninae. However, the arrangement of Euschemoninae as sister to all Hesperiidae (except Coeliadinae), and Eudaminae as sister to Pyrginae, is generally supported by morphology. We suggest that the relationships shown in Figure 1B (also in Figure 3B) should be used as the preferred phylogenetic hypothesis until a better-resolved phylogeny is available.

In addition, we observed unexpected placement of a few taxa within Pyrginae. For instance, Eracon, which was previously classified under Pyrgini (Warren, Ogawa \& Brower, 2009), was found herein to group with Achlyodini. Likewise, Clito grouped within either Pyrgini or Erynnini based on dataset specific analyses. Moreover, we note that both Clito and Eracon sequences in our dataset have $>70 \%$ missing sites. Hence, it is likely that the presence of insufficient informative sites within these taxa might influence their true positions in the phylogeny (Wiens 2003; Wiens and Morrill, 2011). Therefore, for systematic implications, we pruned Clito, Eracon and three additional taxa with a large percentage of missing data from the dataset and reanalysed with gene partitions (Figure 3). We observed no change in tree topology or node support values as a result of pruning these taxa. Hence, although they may appear on the tree in unorthodox positions, it is unlikely that presence of these taxa has any impact on our interpretation of higher-level relationships in the dataset as a whole. corroborate this placement, although its position outside of Eudaminae seems to be correct. 
426 Three tribes within Hesperiinae - Aeromachini, Taractrocerini and Baorini - are monophyletic 427 with high BS values. However, we are uncertain about the phylogenetic status of other proposed 428 tribes within Hesperiinae due to prevalence of low BS values along the short internal branches. 429 This indicates the possible occurrences of rapid ancestral radiation within Hesperiinae and needs 430 further investigation.

431

\section{Conclusions:}

433

434

435

436

437

438

439

440

441

442

443

444

445

446

447

448

449

450

451

452

453

454

455

456

457
With a broad coverage of all known subfamilies, we present the higher level relationships among skipper butterflies. Our analyses suggest possible conflicting topologies with respect to (i) monophyly or paraphyly of Pyrginae and (ii) sister or non-sister status of Eudaminae and Euschemoninae. However none of the topologies resulting from our alternative analyses is strongly supported, and incongruences in signal among genes cannot satisfactorily resolve these differences. We surmise that there is insufficient phylogenetic information in the current dataset to resolve these relationships. It is unlikely that adding data from a few more genes will improve the results, but data from entire genomes may result in a better-resolved phylogeny. However, taking morphological characters into consideration, we suggest one of the topologies as most likely (Figs. 1B \& 3B), and that this topology will aid in future studies on this group.

\section{References:}

Ackery PR. 1984. The Biology of Butterflies. (Eds. Vane-Wright, R. I. \& Ackery, P. R.) 9-21 Academic Press.

Baker RH, DeSalle R. 1997. Multiple sources of character information and the phylogeny of Hawaiian drosophilids. Systematic Biology 46(4):654-673. DOI: 10.1093/sysbio/46.4.654.

Boggs CL, Watt WB, Ehrlich PR (Eds.) 2003. Butterflies: ecology and evolution taking flight. Chicago: University of Chicago Press. 739 pp.

Braby MF, Vila R, Pierce NE. 2006. Molecular phylogeny and systematics of the Pieridae (Lepidoptera: Papilionoidea): higher classification and biogeography. Zoological Journal of the Linnean Society. 147(2):239-275. DOI: 10.1111/j.1096-3642.2006.00218.x.

Bremer K. 1994. Branch support and tree Stability. Cladistics 10(3):295-304. DOI:

10.1111/j.1096-0031.1994.tb00179.x. 
458

459

460

461

462

463

464

465

466

467

468

469

470

471

472

473

474

475

476

477

478

479

480

481

482

483

484

485

486

487

488

489

490

491

Burleigh JG, Mathews S. 2004. Phylogenetic signal in nucleotide data from seed plants: implications for resolving the seed plat tree of life. Am. J. Bot. 91(10): 1599-1613. DOI: 10.3732/ajb.91.10.1599.

Campbell DL, Brower AVZ, Pierce NE. 2000. Molecular evolution of the wingless gene and its implications for the phylogenetic placement of the butterfly family Riodinidae (Lepidoptera: Papilionoidea). Molecular Biology and Evolution 17(5):684-696.

Caterino MS, Reed RD, Kuo MM, Sperling FA. 2001. A partitioned likelihood analysis of swallowtail butterfly phylogeny (Lepidoptera: Papilionidae). Systematic Biology 50(1):106-127. DOI: 10.1080/10635150119988.

Chou, I. 1994. Monographia Rhopalocerorum Sinensium. Henan Scientific and Technological Publishing House.

Chou, I. 1999. Classification and identification of Chinese butterflies. Henan Scientific and Technological Publishing House.

Cummins CA, McInerney JO. 2011. A method for inferring the rate of evolution of homologous characters that can potentially improve phylogenetic inference, resolve deep divergence and correct systematic biases. Systematic Biology 60(6):833-844. DOI:

10.1093/sysbio/syr064.

Dunn CW, Hejnol A, Matus DQ, Pang K, Browne WE, Smith SA, Seaver E, Rouse GW, Obst M, Edgecombe GD, Sorensen MV, Haddock SHD, Schmidt-Rhaesa A, Okusu A, Kristensen RM, Wheeler WC, Martindale MQ, Giribet G. 2008. Broad phylogenomic sampling improves resolution of the animal tree of life. Nature 452(7188):745-9. doi:10.1038/nature06614.

Eckert AJ, Carstens BC. 2008. Does gene flow destroy phylogenetic signal? The performance of three methods for estimating species phylogenies in the presence of gene flow. Molecular Phylogenetics and Evolution 49(3): 832-842. DOI: 10.1016/j.ympev.2008.09.008.

Espeland M, Hall JP, DeVries PJ, Lees DC, Cornwall M, Hsu YF, Wu LW, Campbell DL, Talavera G, Vila R, Salzman S, Ruehr S, Lohman DJ, Pierce N. 2015. Ancient Neotropical origin and recent recolonisation: Phylogeny, biogeography and diversification of the Riodinidae (Lepidoptera: Papilionoidea). Molecular Phylogenetics and Evolution 93:296-306. DOI: 10.1016/j.ympev.2015.08.006.

Evans WH. 1949. A catalogue of the Hesperiidae from Europe, Asia, and Australia in the British Museum (Natural History). British Museum, London.

Felsenstein J. 1985. Phylogenies and the comparative method. The American Naturalist 125(1):1-15. 
492 Gelman A, Rubin DB. 1992. Inference from iterative simulation using multiple

493 sequences. Statistical Science 7(4):457-472.

494 Goloboff PA. 1999. Analyzing large data sets in reasonable times: solutions for composite

495 optima. Cladistics 15(4):415-428. DOI: 10.1111/j.1096-0031.1999.tb00278.x.

496

497

498

499

500

501

502

503

504

505

506

507

508

509

510

511

512

513

514

515

516

517

518

519

520

521

522

523

524

525

Goloboff PA, Farris JS, Nixon KC. 2008. TNT, a free program for phylogenetic analysis. Cladistics 24(5):774-786. DOI: 10.1111/j.1096-0031.2008.00217.x.

Heikkila M, Kaila L, Mutanen M, Pena C, Wahlberg N. 2012. Cretaceous origin and repeated tertiary diversification of the redefined butterflies. Proceedings of the Royal Society B: Biological Sciences 279(1731):1093-1099. DOI: 10.1098/rspb.2011.1430.

Hernández-Roldán JL, Bofill R, Dapporto L, Munguira ML, Vila R. 2014. Morphological and chemical analysis of male scent organs in the butterfly genus Pyrgus (Lepidoptera: Hesperiidae). Organisms Diversity \& Evolution 14(3):269-278. DOI: 10.1007/s13127014-0170-x.

Huelsenbeck JP, Larget B, Alfaro ME. 2004. Bayesian phylogenetic model selection using reversible jump markov chain monte carlo. Mol. Biol. Evol. 21(6):1123-1133.

Jarvis ED, Mirarab S, Aberer AJ, Li B, Houde P, Li C, ... \& Suh A. 2014. Whole-genome analyses resolve early branches in the tree of life of modern birds. Science 346(6215):1320-31. DOI: 10.1126/science.1253451.

Johnson Johnson BR, Borowiec ML, Chiu JC, Lee EK, Atallah J, Ward PS. 2013. Phylogenomics resolves evolutionary relationships among ants, bees, and wasps. Curr Biol. 23:2058-062. doi:10.1016/j.cub.2013.08.050.

Kim MJ, Wang AR, Park JS, Kim I. 2014. Complete mitochondrial genomes of five skippers (Lepidoptera: Hesperiidae) and phylogenetic reconstruction of Lepidoptera. Gene 549(1):97-112. DOI: 10.1016/j.gene.2014.07.052.

Kocot KM, Cannon JT, Todt C, Citarella MR, Kohn AB, Meyer A, Santos SR, Schander C, Moroz LL, Lieb B, Halanych KM. 2011. Phylogenomics reveals deep molluscan relationships. Nature 477:452-6. doi:10.1038/nature10382.

Kodandaramaiah U, Lees DC, Müller CJ, Torres E, Karanth KP, Wahlberg N. 2010.

Phylogenetics and biogeography of a spectacular Old World radiation of butterflies: the subtribe Mycalesina (Lepidoptera: Nymphalidae: Satyrini). BMC Evolutionary Biology 10(1):172. DOI: 10.1186/1471-2148-10-172.

Lanfear R, Calcott B, Ho SYW, Guindon S. 2012. PartitionFinder: Combined Selection of Partitioninggsdhjh Schemes and Substitution Models for Phylogenetic Analyses. Molecular Biology and Evolution 29:1695-1701. 
526 Lento GM, Hickson RE, Chambers GK, Penny D. 1995. Use of spectral analysis to test

527

528

529

530

531

532

533

534

535

536

537

538

539

540

541

542

543

544

545

546

547

548

549

550

551

552

553

554

555

556

557

558 hypotheses on the origin of pinnipeds. Molecular biology and evolution 12(1):28-52. DOI: 10.1093/oxfordjournals.molbev.a040189.

Mielke OHH. 2005. Catalogue of the American Hesperioidea: Hesperiidae (Lepidoptera). Sociedade Brasileira de Zoologia, Parana, Brazil. 6 vols.

Miller MA, Pfeiffer W, Schwartz T. 2010. Creating the CIPRES Science Gateway for inference of large phylogenetic trees. In: Proceedings of the Gateway Computing Environments Workshop (GCE), 14 Nov. 2010, New Orleans, LA: 1 - 8.

Nabholz B, Kunstner A, Wang R, Jarvis ED, Ellegren H. 2011. Dynamic evolution of base composition: causes and consequences in avian phylogenomics. Molecular Biology and Evolution 28(8):2197-2210. DOI: 10.1093/molbev/msr047.

Nazari V, Zakharov EV, Sperling FA. 2007. Phylogeny, historical biogeography and taxonomic ranking of Parnassiinae (Lepidoptera, Papilionidae) based on morphology and seven genes. Molecular Phylogenetics and Evolution 42(1):131-156. DOI: 10.1016/j.ympev.2006.06.022.

Nixon KC. 1999. The parsimony ratchet, a new method for rapid parsimony analysis. Cladistics 15(4):407-414. DOI: 10.1111/j.1096-0031.1999.tb00277.x.

Planet PJ. 2006. Tree disagreement: measuring and testing incongruence in phylogenies. Journal of Biomedical Informatics 39(1):86-102. DOI: 10.1016/j.jbi.2005.08.008.

Pollard DA, Iyer VN, Moses AM, Eisen MB. 2006. Widespread discordance of gene trees with species tree in Drosophila: evidence for incomplete lineage sorting. PLoS Genet 2(10):e173. DOI: 10.1371/journal.pgen.0020173.

Rambaut A, Suchard MA, Xie D, Drummond AJ. 2014. Tracer v1.6. Available at http://beast.bio.ed.ac.um/Tracer.

Regier JC, Shultz JW, Ganley AR, Hussey A, Shi D, Ball B, Zwick A, Stajich JE, Cummings MP, Martin JW, Cunningham CW. 2008. Resolving arthropod phylogeny: exploring phylogenetic signal within $41 \mathrm{~kb}$ of protein-coding nuclear gene sequence. Systematic Biology 57(6):920-938. DOI: 10.1080/10635150802570791.

Regier JC, Shultz JW, Zwick A, Hussey A, Ball B, Wetzer R, Martin JW, Cunningham CW. 2010. Arthropod relationships revealed by phylogenomic analysis of nuclear proteincoding sequences. Nature 463(7284):1079-1083. DOI: 10.1038/nature08742.

Richart CH, Hayashi CY, Hedin M. 2016. Phylogenomic analyses resolve an ancient trichotomy at the base of Ischyropsalidoidea (Arachnida, Opiliones) despite high levels of gene tree 
559

560

561

562

563

564

565

566

567

568

569

570

571

572

573

574

575

576

577

578

579

580

581

582

583

584

585

586

587

588

589

590

591

conflict and unequal minority resolution frequencies. Mol. Phyl. Evol. 95:171-182. DOI: 10.1016/j.ympev.2015.11.010.

Rokas A, King N, Finnerty J, Carroll SB. 2003. Conflicting phylogenetic signals at the base of the metazoan tree. Evolution and Development 5(4):346-359. DOI: 10.1046/j.1525142X.2003.03042.x.

Rokas A, Williams BL, King N, Carroll SB. 2003. Genome-scale approaches to resolving incongruence in molecular phylogenies. Nature 425(6960):798-804. DOI: 10.1038 /nature 02053 .

Ronquist F, Teslenko M, van der Mark P, Ayres DL, Darling A, Höhna S, Larget B, Liu L, Suchard MA, Huelsenbeck JP. 2012. MrBayes 3.2: efficient Bayesian phylogenetic inference and model choice across a large model space. Systematic biology 61(3):539-42. DOI: $10.1093 /$ sysbio/sys029.

Rota, J. \& Wahlberg, N. 2012. Exploration of data partitioning in an eight-gene dataset: phylogeny of metalmark moths (Lepidoptera, Choreutidae). Zoologica Scripta 41: 536-546. doi:10.1111/j.1463-6409.2012.00551.x.

Ruhfel BR, Gitzendanner MA, Soltis PS, Soltis DE, Burleigh JG. 2014. From algae to angiosperms - inferring the phylogeny of green plants (Viridiplantae) from 360 plastid genomes. BMC Evol. Biol. 14:23. DOI: 10.1186/1471-2148-14-23.

Scott JA. 1985. The phylogeny of butterflies (Papilionoidea and Hesperioidea). J. Res. Lepidoptera 23(4):241-281.

Scott JA \& Wright DM. 1990. Butterflies of Europe (eds Kudrna, O.). Aula-Verlag.

Shimodaira H. 2002. An approximately unbiased test of phylogenetic tree selection. Systematic Biology 51(3):492-508. DOI: 10.1080/10635150290069913.

Simonsen TJ, Zakharov EV, Djernaes M, Cotton AM, Vane-Wright RI, Sperling FA. 2011. Phylogenetics and divergence times of Papilioninae (Lepidoptera) with special reference to the enigmatic genera Teinopalpus and Meandrusa. Cladistics 27(2):113-137. DOI: 10.1111/j.1096-0031.2010.00326.x.

Smith SA, Wilson NG, Goetz FE, Feehery C, Andrade SCS, Rouse GW, Giribet G, Dunn CW. 2011. Resolving the evolutionary relationships of molluscs with phylogenomic tools. Nature 480:364-7. doi:10.1038/nature10526.

Smith SA, Moore MJ, Brown JW and Yang Y. 2015. Analysis of phylogenomic datasets reveals conflict, concordance, and gene duplications with examples from animals and plants. BMC Evol. Biol. 15:150. DOI: 10.1186/s12862-015-0423-0. 
592 Soltis DE, Soltis PS, Zanis MJ. 2002. Phylogeny of seed plants based on evidence from eight 593 genes. Am. J. Bot. 89(10): 1670-1681. DOI: 10.3732/ajb.89.10.1670.

594 Song S, Liu L, Edwards SV, Wu S. 2012. Resolving conflict in eutherian mammal phylogeny 595 596 using phylogenomics and the multispecies coalescent model. Proc. Nat. Acad. Sci. 109(37):14942-14947.

Stamatakis A. 2014. RAxML version 8: a tool for phylogenetic analysis and post-analysis of large phylogenies. Bioinformatics 30:1312-1313. DOI: 10.1093/bioinformatics/btu033.

Voss EG. 1952. On the classification of the Hesperiidae. Annals of the Entomological Society of America 45(2):246-258. DOI: 10.1093/aesa/45.2.246.

601

602

603

604

605

606

607

608

609

610

611

612

613

614

615

616

617

618

619

620

621

622

623

Wahlberg N, Leneveu J, Kodandaramaiah U, Pena C, Nylin S, Freitas AVL, Brower AVZ. 2009. Nymphalid butterflies diversify following near demise at the Cretaceous/Tertiary boundary. Proceedings of the Royal Society B: Biological Sciences 276:4295-4320. DOI: 10.1098/rspb.2009.1303.

Wahlberg N, Rota J, Braby MF, Pierce NE, Wheat CW. 2014. Revised systematics and higher classification of pierid butterflies (Lepidoptera: Pieridae) based on molecular data. Zoologica Scripta 43(6):641-650. DOI: 10.1111/zsc.12075.

Wahlberg N, Wheat CW. 2008. Genomic outposts serve the phylogenomic pioneers: designing novel nuclear markers for genomic DNA extractions of Lepidoptera. Systematic Biology 57(2):231-242. DOI: 10.1080/10635150802033006.

Warren AD, Ogawa JR, Brower AVZ. 2008. Phylogenetic relationships of subfamilies and circumscription of tribes in the family Hesperiidae (Lepidoptera: Hesperioidea). Cladistics 24(5):642-676. DOI: 10.1111/j.1096-0031.2008.00218.x.

Warren AD, Ogawa JR, Brower AVZ. 2009. Revised classification of the family Hesperiidae (Lepidoptera: Hesperioidea) based on combined molecular and morphological data. Systematic Entomology 34(3):467-523. DOI: 10.1111/j.1365-3113.2008.00463.x.

Whitfield JB, Lockhart PJ. 2007. Deciphering ancient rapid radiations. Trends in Ecology \& Evolution 22(5):258-265. DOI: 10.1016/j.tree.2007.01.012.

Wiens, JJ. 2003. Missing data, incomplete taxa, and phylogenetic accuracy. Systematic Biology 52(4):528-538. DOI: 10.1080/10635150390218330.

Wiens, JJ and Morrill MC. 2011. Missing data in phylogenetic analysis: reconciling results from simulations and empirical data. Systematic Biology 60(5):719-731. DOI: $10.1093 /$ sysbio/syr025. 
624 Wolf YI, Rogozin IB, Grishin NV, Koonin EV. 2002. Genome trees and the tree of life. Trends 625 in Genetics 18(9):472-479. DOI: 10.1016/S0168-9525(02)02744-0.

626 Xi Z, Liu L, Rest JS, Davis CC. 2014. Coalescent versus concatenation methods and the 627 628 placement of amborella as sister to water lilies. Systematic Biology 63(6): 919-932. DOI: 10.1093/sysbio/syu055.

Xia X. 2013. DAMBE5: A comprehensive software package for data analysis in molecular biology and evolution. Mol Biol Evol 30(7): 1720-1728. DOI: 10.1093/molbev/mst064.

631 Yuan X, Gao K, Yuan F, Wang P, Zhang Y. 2015. Phylogenetic relationships of subfamilies in 632 the family Hesperiidae (Lepidoptera: Hesperioidea) from China. Scientific Reports 5:11140. DOI: $10.1038 /$ srep 11140 .

634 Zwick A, Reiger JC, Zwickl DJ. 2012. Resolving discrepancy between nucleotides and amino 635 acids in deep-level arthropod phylogenomics: differentiating serine codons in 21-amino acid models. PLoS ONE 7(11):e47450. DOI: 10.1371/journal.pone.0047450. 


\section{Figure 1}

Comparison of best ML trees across analyses.

The best ML trees from the concatenated dataset (A), combined-nuclear dataset (B) and degenerated dataset (degen_1) (C). The values at nodes in (A) and (B) represent support from 1000 bootstrap trees analyzed with TIGER-partitions / gene-partitions. In (C), the values at the nodes are the output from the analyses using TIGER-partitions only and the nodes without any value have bootstrap support $<20$. The other degenerated datasets (nt_12, noLRall1+nt2) have similar or more fluctuating rearrangements for the nodes with $B S<20$.

*Note: Auto Gamma Correction was used for the image. This only affects the reviewing manuscript. See original source image if needed for review.

A

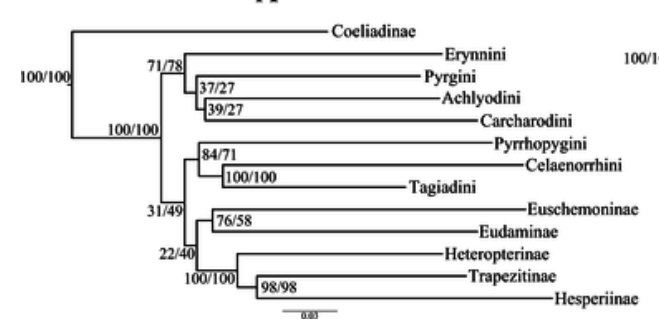

B

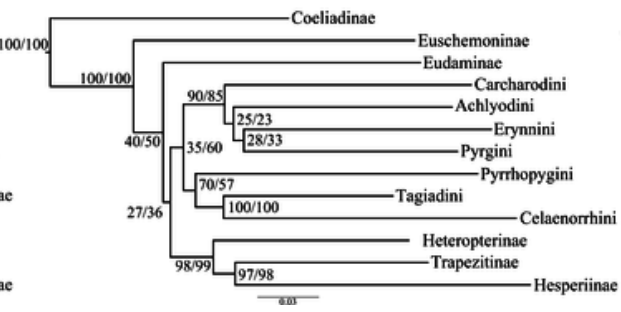

$\mathrm{C}$

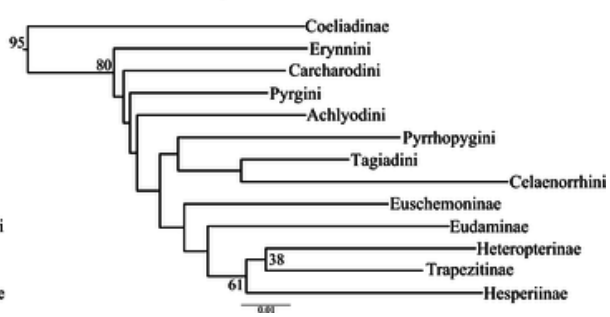


Figure 2

Comparison of the supports/conflicts on the Lento plots.

The Lento plots were drawn from $105 \mathrm{ML}$ trees recovered during best ML tree search across different datasets using two different partitioning schemes. The X-axis represents each nontrivial clade, with filled circles indicating the clade composition; the $\mathrm{Y}$-axis shows relative support (values above zero) or conflict (values below zero). Splits are colour coded to aid comparison across analyses. The splits which are not coloured are found only in that particular analysis and not recovered from others.

Nuclear dataset

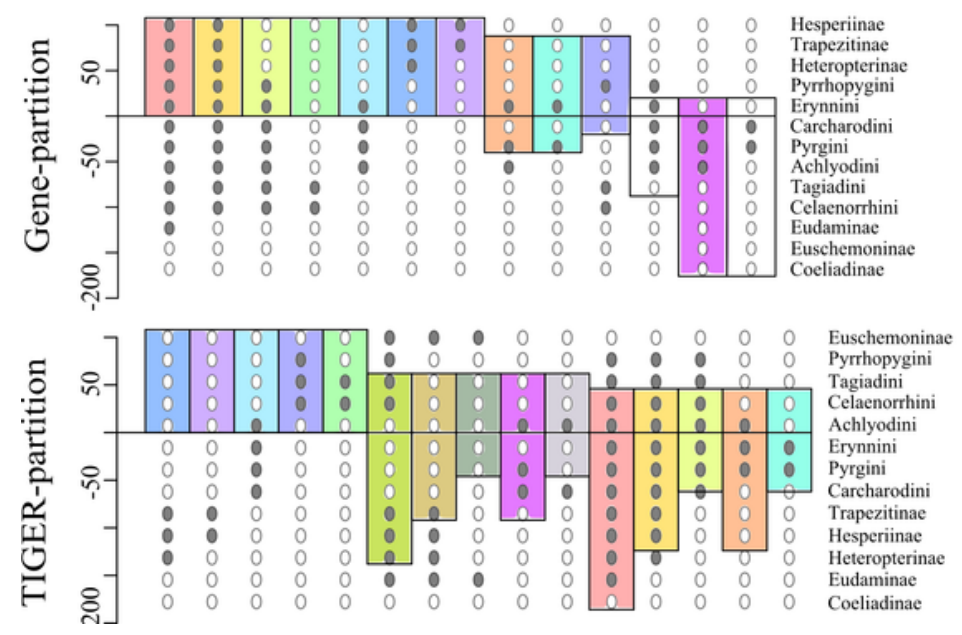

Concatenated dataset
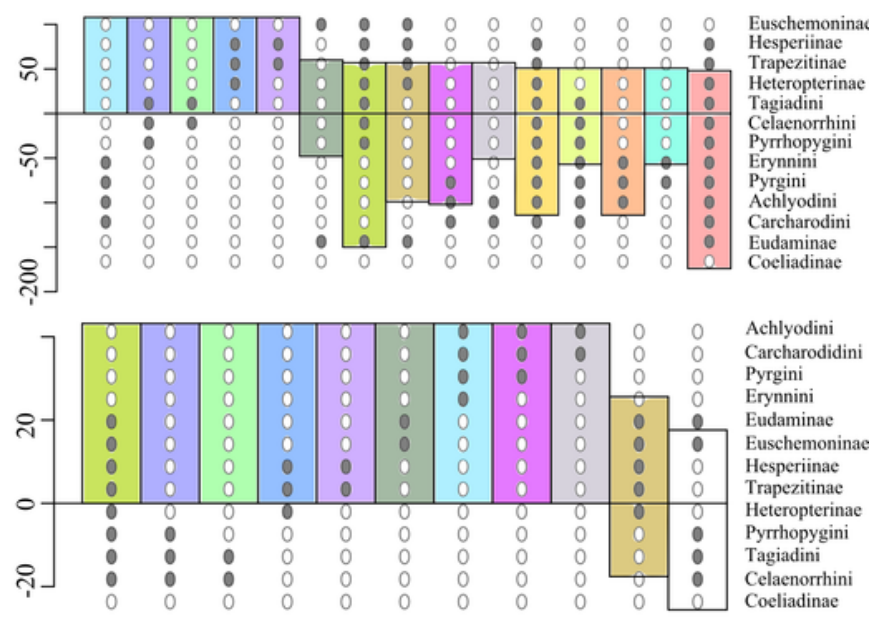


\section{Figure 3}

The ML trees from a reduced dataset.

The ML trees from the analyses of (A) the concatenated dataset with gene partitions, and (B) the combined nuclear dataset with gene partitions. Clito, Eracon and three additional taxa were removed prior to the analyses (see text for detail). The size of the circle at the node corresponds to the bootstrap support that was derived from 1000 pseudo-replicates. All taxa are colour coded based on their subfamily status, except the taxa within subfamily Pyrginae which are coloured based on their tribe. Silhouette $\odot$ PhyloPic. 

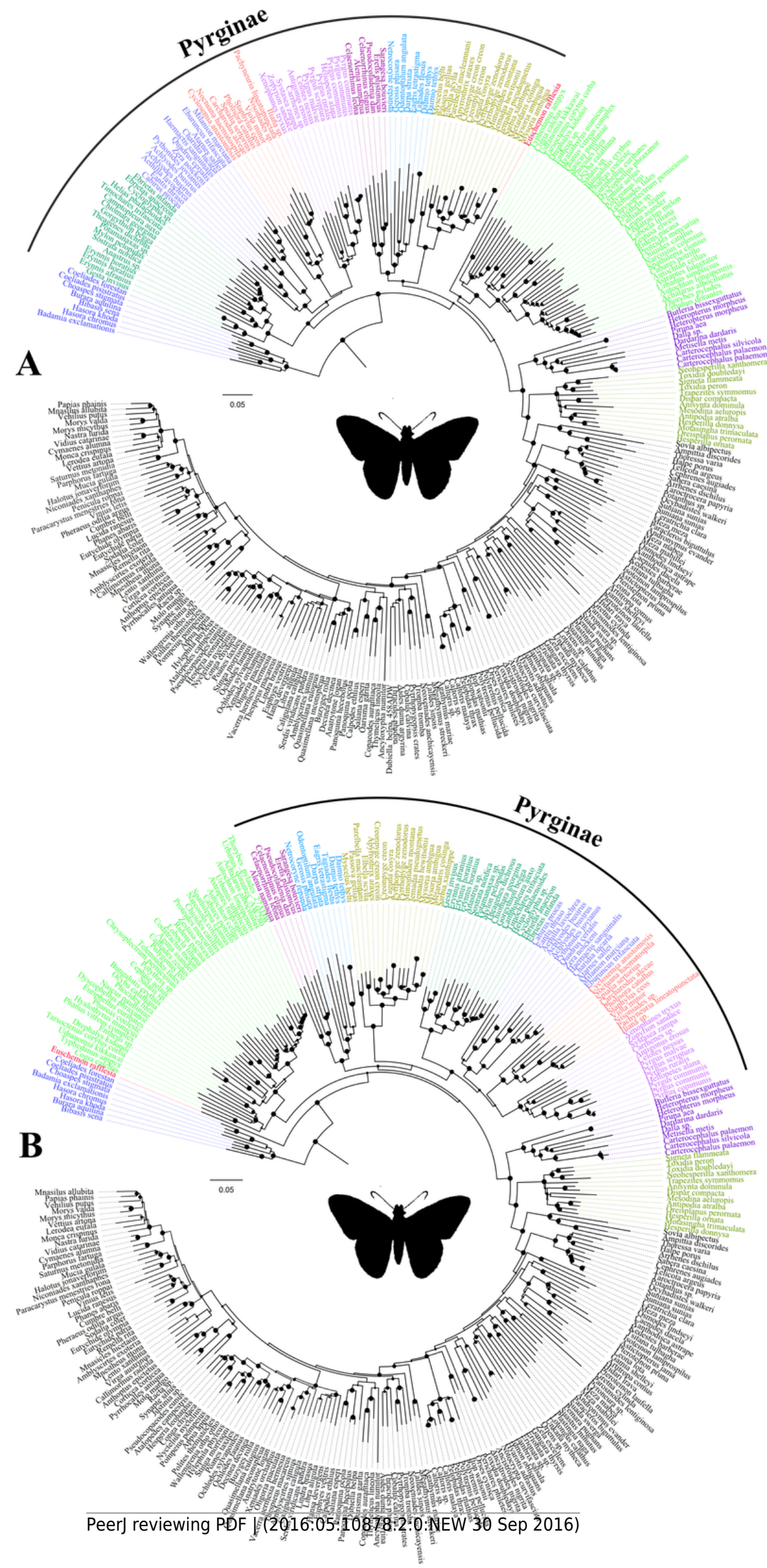